\title{
ON THE CRITICAL PERIODS OF LIÉNARD SYSTEMS WITH CUBIC RESTORING FORCES
}

\section{ZHENGDONG DU}

Received 23 February 2004

\begin{abstract}
We study local bifurcations of critical periods in the neighborhood of a nondegenerate center of a Liénard system of the form $\dot{x}=-y+F(x), \dot{y}=g(x)$, where $F(x)$ and $g(x)$ are polynomials such that $\operatorname{deg}(g(x)) \leq 3, g(0)=0$, and $g^{\prime}(0)=1, F(0)=F^{\prime}(0)=0$ and the system always has a center at $(0,0)$. The set of coefficients of $F(x)$ and $g(x)$ is split into two strata denoted by $S_{I}$ and $S_{I I}$ and $(0,0)$ is called weak center of type $I$ and type $I I$, respectively. By using a similar method implemented in previous works which is based on the analysis of the coefficients of the Taylor series of the period function, we show that for a weak center of type $I$, at most $[(1 / 2) \operatorname{deg}(F(x))]-1$ local critical periods can bifurcate and the maximum number can be reached. For a weak center of type $I I$, the maximum number of local critical periods that can bifurcate is at least $[(1 / 4) \operatorname{deg}(F(x))]$.
\end{abstract}

2000 Mathematics Subject Classification: 34C23, 37G10.

1. Introduction. During the last decades, there has been considerable interest in studying the generalized Liénard system of the form

$$
\dot{x}=-y+F(x), \quad \dot{y}=g(x),
$$

or its equivalent form

$$
\dot{x}=-y, \quad \dot{y}=g(x)+f(x) y,
$$

where $f(x)=F^{\prime}(x)$. The popularity is due to at least two reasons. First, it generalizes many oscillation systems arising from applications. Second, many other systems can be transformed into the form (1.1) or (1.2) (see [1]).

One of the most studied problems is to determine the number and relative configuration of limit cycles of (1.1) in terms of the properties of $F(x)$ and $g(x)$. There is an enormous literature on this problem, see, for example, [15] for more on these issues. For the special class of system (1.2), where $f(x)$ and $g(x)$ are polynomials of degrees at most $n$ and $m$, respectively, there are also extensive studies of the cyclicity $\hat{H}_{n, m}$, that is, the maximum number of small amplitude limit cycles bifurcating from the fine focus of (1.2). In [7], Christopher and Lynch give results for $\hat{H}_{n, m}$ when $f(x)$ or $g(x)$ is quadratic or cubic polynomial.

The purpose of this paper is to examine the local bifurcations of critical periods in the neighborhood of a nondegenerate center of system (1.1), where $F(x)$ and $g(x)$ are polynomials such that $\operatorname{deg}(g(x)) \leq 3, g(0)=0$, and $g^{\prime}(0)=1, F(0)=F^{\prime}(0)=0$ and 
the system always has a center at $(0,0)$. Let $G(x)=\int_{0}^{x} g(\xi) d \xi$, the center condition for polynomial Liénard systems is given by the following theorem of Christopher (see [5]).

THEOREM 1.1 [5]. If $F(x)$ and $g(x)$ are polynomials, then system (1.1) has a nondegenerate center at $(0,0)$ if and only if $F(x)$ and $G(x)$ are both polynomials of a polynomial $M(x)$ with $M(0)=M^{\prime}(0)=0$ and $M^{\prime \prime}(0) \neq 0$.

The bifurcation of critical periods from centers of planar vector fields is an important problem, because it is closely related to the monotonicity of periods of closed orbits surrounding a center and subharmonic bifurcation for periodically forced systems. Similar to Hilbert's 16th problem, the following problem can be formulated.

Problem 1.2. Determine the maximum number $\mathscr{b}(n)$ of critical periods of polynomial systems of degree $n$ with nondegenerate centers in terms of $n$ only.

While Problem 1.2 is still completely open, an easier problem is proposed.

Problem 1.3. Determine the maximum number $\tilde{\mathscr{C}}(n)$ of local critical periods bifurcating from a weak center of polynomial systems of degree $n$ in terms of $n$ only.

In 1989, Chicone and Jacobs (see [3]) developed a general theory of solving Problem 1.3 and proved that $\tilde{\mathscr{C}}(2)=2$. However, the problem for higher-degree systems is still unsolved. A few classes of cubic systems studied in $[10,11,13,14]$ proved that $\tilde{\mathscr{C}}(3) \geq 4$. It is worth noting that some researchers have considered the global problem of bifurcations of critical periods for some specific systems, see, for example, [9]. However, there is still no general method of solving Problem 1.2.

The monotonicity of the period function of centers of system (1.2) or isochronicity has been studied by several authors (see [12] and the references therein). Recently, Christopher and Devlin [6] gave a complete classification for isochronous centers of polynomial Liénard systems of degree 34 or less. However, there are only very few studies on the number of critical periods that can bifurcate from the nondegenerate center of (1.1) for the special case where $F(x) \equiv 0$, which significantly simplifies matters (see $[3,4])$.

Under our assumption, $\operatorname{deg}(G(x)) \leq 4$ and by Theorem 1.1, both $F(x)$ and $G(x)$ are polynomials of $M(x)$, where $M(x)$ is as in Theorem 1.1. Without loss of generality, we assume that $M^{\prime \prime}(0)=2$. Thus, $M(x)=x^{2}+O\left(x^{3}\right)$ and $\operatorname{deg}(M(x)) \leq 4$. So, we write $M(x)=x^{2}+b_{1} x^{3}+b_{2} x^{4}$ and $F(x), G(x)$ have the form

$$
F=\sum_{k=1}^{n} a_{k} M^{k}, \quad G=\frac{1}{2} M+b_{0} M^{2}
$$

Clearly, there are only 2 possibilities: $b_{0}=0$ or $b_{0} \neq 0$ and $M(x)=x^{2}$. To unify these two cases, we write system (1.1) into the following form:

$$
\dot{x}=-y+\sum_{k=1}^{n} a_{k}\left(x^{2}+b_{1} x^{3}+b_{2} x^{4}\right)^{k}, \quad \dot{y}=x+\frac{3}{2} b_{1} x^{2}+\left(b_{0}+2 b_{2}\right) x^{3} .
$$


Thus, $F(x)$ is as in (1.3) and $g(x), G(x)$ are given below:

$$
g(x)=x+\frac{3}{2} b_{1} x^{2}+\left(b_{0}+2 b_{2}\right) x^{3}, \quad G(x)=\frac{1}{2} M(x)+\frac{1}{4} b_{0} x^{4} .
$$

For the coefficients $\left(b_{0}, b_{1}, b_{2}, a_{1}, \ldots, a_{n}\right)$ we will use the abbreviation $\lambda$ so that $\lambda_{k}=b_{k}$ for $k=0,1,2$ and $\lambda_{k+2}=a_{k}$ for $k=1, \ldots, n$. In particular, we have $\lambda \in \mathbb{R}^{n+3}$. Then, from Theorem 1.1 we have the following lemma.

LEMMA 1.4. System (1.4) has a nondegenerate center at $(0,0)$ if and only if the parameter value $\lambda \in \mathbb{R}^{n+3}$ is in one of the following strata:

$$
S_{I}:=\left\{\lambda \in \mathbb{R}^{n+3} \mid \lambda_{0} \neq 0, \lambda_{1}=\lambda_{2}=0\right\}, \quad S_{I I}:=\left\{\lambda \in \mathbb{R}^{n+3} \mid \lambda_{0}=0\right\} .
$$

We say system (1.4) has a weak center of type I (resp., type II) if the system is nonlinear and $\lambda \in S_{I}$ (resp., $\lambda \in S_{I I}$ ). Our main result is the following theorem.

THEOREM 1.5. (1) System (1.4) has an isochronous center at the origin if and only if $b_{0}=(4 / 9) a_{0}^{2}$ and $b_{1}=b_{2}=a_{1}=\cdots=a_{n}=0$. At most $n-1$ critical periods can bifurcate from the nonlinear isochronous center and there are perturbations to produce $n-1$ critical periods.

(2) If system (1.4) has a weak center of type I of finite order at the origin, then at most $n-1$ critical periods can bifurcate from the weak center and there are perturbations to produce $n-1$ critical periods.

(3) The maximum critical periods can bifurcate from a weak center of type II of system (1.4) is at least $n$.

It is clear from Theorem 1.5 that there are no nonlinear isochronous centers inside type $I I$. For a weak center of type $I, \operatorname{deg}(F(x))=2 n$, the maximum local critical periods that can bifurcate are $[(1 / 2) \operatorname{deg}(F(x))]-1$ and this upper bound can be attained. For a weak center of type $I I, \operatorname{deg}(F(x))=4 n$, the maximum local critical periods can bifurcate is at least $[(1 / 4) \operatorname{deg}(F(x))]$.

Our approach is similar to the one implemented in [3, 10, 11, 13]. It is based on the analysis of the coefficients of the Taylor series of the period function. The Taylor coefficients of the period function have been computed and simplified by reduction modulo a Gröbner basis using Maple for low degrees of $F(x)$. This enables us to conjecture a general pattern for the ideal generated by the coefficients over the polynomial ring of the parameters. These conjectures are then proved rigorously using arguments similar to those used by Bautin in [2] to determine the structure of ideal generated by focal values of quadratic system.

This paper is organized as follows. Section 2.1 summarizes the general results by Chicone and Jacobs (see [3]). Section 2.2 summarizes a recursive algorithm to compute the period coefficients. Section 3 considers weak center of type $I$. Section 4 considers weak center of type II. Section 5 is the proof of Theorem 1.5. 


\section{Preliminary}

2.1. Local critical periods of polynomial systems. Consider a family of planar ordinary differential systems with a nondegenerate center at the origin

$$
\dot{x}=-y+p(x, y, v), \quad \dot{y}=x+q(x, y, v),
$$

where $p(x, y, v)$ and $q(x, y, v)$ are polynomials of degree $n$ in $x$ and $y$, the parameter $v=\left(v_{1}, \ldots, v_{m}\right) \in \mathbb{R}^{m}$. The minimum period $T(r, v)$ of the periodic orbit passing through $(r, 0)$ associated with the center of the above system for the parameter $v$ yields the so-called period function $T$. Let $P(r, v)=T(r, v)-2 \pi$. For $v_{*} \in \mathbb{R}^{m}$, the origin is called a weak center of finite order $k$ if $P\left(0, v_{*}\right)=P^{\prime}\left(0, v_{*}\right)=\cdots=P^{(2 k+1)}\left(0, v_{*}\right)=0$ and $P^{(2 k+2)}\left(0, v_{*}\right) \neq 0$, where the derivatives are taken with respect to $r$. The origin is called an isochronous center if $P^{(k)}\left(0, v_{*}\right)=0$ for all $k \geq 0$. A critical period is a period corresponding to a solution of the equation $P_{r}(r, v)=0$ as $v$ varies. A local critical period is a period corresponding to a critical point of $P(r, v)$ which arises from a bifurcation from a weak center.

For $v_{*}$ corresponding to a weak center, the function $(r, v) \mapsto T(r, v)$ is analytic in a neighborhood of $\left(0, v_{*}\right)$ and can be represented by its Taylor series

$$
T(r, v)=2 \pi+\sum_{k=2}^{\infty} p_{k}(v) r^{2 k}
$$

for $|r|$ and $\left|v-v_{*}\right|$ sufficiently small. Here, the period coefficients $p_{k} \in \mathbb{R}\left[v_{1}, \ldots, v_{m}\right]$, the Noetherian ring of polynomials in the variables $v_{1}, \ldots, v_{m}$ and for any $k \geq 1, p_{2 k+1} \in$ $\left(p_{2}, p_{4}, \ldots, p_{2 k}\right)$, the ideal generated by $p_{2}, p_{4}, \ldots, p_{2 k}$. In particular, for any $v$, the first $k \geq 1$ such that $p_{k}(v) \neq 0$ is even (see [3]). The theory of Chicone and Jacobs in [3] is based on the analysis of the period coefficients. To state their theorems precisely, we first introduce the following concept.

DEFINITION 2.1 [11]. Let $\left\{\chi_{v}\right\}_{v \in \mathbb{R}^{m}}$ be a family of systems with a center at the origin and associated period coefficients $p_{2 k}(v)$. The family is said to satisfy condition $(\mathscr{P})$ if for any $v_{*} \in V\left(p_{2}, p_{4}, \ldots, p_{2 k}\right):=\left\{v \mid p_{2 i}(v)=0, i=1, \ldots, k\right\}, p_{2 k+2}\left(\nu_{*}\right) \neq 0$ and any neighborhood $W \subset \mathbb{R}^{m}$ of $\nu_{*}$ in which $p_{2 k+2} \neq 0$, there exists $v^{\prime} \in W$ such that

$$
p_{2 k}\left(v^{\prime}\right) p_{2 k+2}\left(v^{\prime}\right)<0, \quad \text { with } v^{\prime} \in V\left(p_{2}, p_{4}, \ldots, p_{2 k-2}\right) \text {. }
$$

The system $\chi_{v_{*}}$ is said to satisfy condition $\left(\mathscr{P}_{k}\right)$.

The following version of the theorems of Chicone and Jacobs [3] are given by Rousseau and Toni in [11].

FINITE-ORDER BIFURCATION THEOREM. From weak centers of finite order $k$ at the parameter value $v_{*}$, no more than $k$ local critical periods bifurcate. Moreover, if the family satisfies the condition $(\mathscr{P})$ and if $\chi_{v_{*}}$ satisfies the condition $\left(\mathscr{P}_{k}\right)$, then there are perturbations with exactly $j$ local critical periods for any $0 \leq j \leq k$. 
ISOCHRONE BIFURCATION THEOREM. If the vector field (2.1) has an isochronous center at the origin for the parameter value $v_{*}$ and if for each integer $n \geq 1$, the period coefficient $p_{2 n}$ is in the ideal $\left(p_{2}, p_{4}, \ldots, p_{2 k}, p_{2 k+2}\right)$ over the ring $\mathbb{R}\left\{v_{1}, \ldots, v_{m}\right\}_{\nu_{*}}$ of convergent power series at $v_{*}$, then at most $k$ local critical periods bifurcate from the isochronous

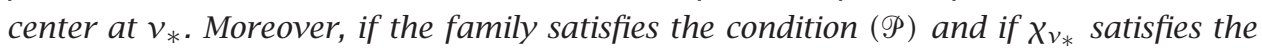
condition $\left(\mathscr{P}_{k}\right)$, then exactly $j$ local critical periods bifurcate from the center at $v_{*}$ for any $0 \leq j \leq k$.

2.2. The computation of the periodic coefficients. Let $g_{0}(x)=g(x)-x$ and transform (1.4) to polar coordinates by $x=r \cos \theta, y=r \sin \theta$, and eliminating time yields

$$
\frac{d r}{d \theta}=\frac{F(r \cos \theta) \cos \theta+g_{0}(r \cos \theta) \sin \theta}{1+(1 / r) g_{0}(r \cos \theta) \cos \theta-(1 / r) F(r \cos \theta) \sin \theta}
$$

Then, (2.4) is analytic and we assume the following expansion:

$$
\frac{d r}{d \theta}=\sum_{k=2}^{\infty} A_{k}(\theta) r^{k}, \quad \frac{d t}{d \theta}=\sum_{k=0}^{\infty} B_{k}(\theta) r^{k}
$$

where $B_{0}(\theta) \equiv 1$. Let $\gamma \xi$ be the closed orbit of (1.4), through $(\xi, 0)$. The period function is given by

$$
T(\xi, \lambda)=\int_{\gamma \xi} d t=\int_{0}^{2 \pi} \frac{d t}{d \theta} d \theta=2 \pi+\int_{0}^{2 \pi} \sum_{k=1}^{\infty} B_{k}(\theta) r^{k} d \theta,
$$

where $r=r(\theta, \xi, \lambda)$ is the solution of (1.4), with the initial condition $r(0, \lambda)=\xi . r(\theta, \xi, \lambda)$ may be locally represented as a convergent power series in $\xi$ :

$$
r(\theta, \xi, \lambda)=\sum_{k=1}^{\infty} u_{k}(\theta, \lambda) \xi^{k}
$$

where $u_{1}(0, \lambda)=1$ and $u_{k}(0, \lambda)=0$ for any $k>1$ and $\lambda$. Substituting (2.7) into (2.5) and comparing the coefficients of $\xi^{k}, k \geq 1$, we obtain recursive equations for $u_{k}$. For example, the first 3 equations are given by

$$
\frac{d u_{1}}{d \theta}=0, \quad \frac{d u_{2}}{d \theta}=A_{2} u_{1}^{2}, \quad \frac{d u_{3}}{d \theta}=A_{3} u_{1}^{3}+2 A_{2} u_{1} u_{2},
$$

which can be found by direct integration. From (2.6) and (2.7), we obtain a recursive algorithm for computing the period coefficients $p_{2}, p_{4}, \ldots, p_{2 k}, \ldots$. The algorithm can be easily implemented in the computer algebra system Maple.

3. Weak center of type $I$. A weak center of type $I$ corresponds to the case where $F(x)$ is a polynomial of degree $2 n$ and system (1.4) has the following form:

$$
\dot{x}=-y+\sum_{k=1}^{n} a_{k} x^{2 k}, \quad \dot{y}=x+b_{0} x^{3},
$$

where $b_{0} \neq 0$. Direct computation with the aid of Maple yields the following lemma. 
LEMMA 3.1. The period coefficient $p_{2}$ for (3.1) is given by $p_{2}=(\pi / 12)\left(4 a_{1}^{2}-9 b_{0}\right)$.

It is convenient to split the set $S_{I}$ as $S_{I}=S_{I}^{A} \cup S_{I}^{B}$, where

$$
\begin{aligned}
& S_{I}^{A}:=\left\{\lambda \in \mathbb{R}^{n+3} \mid \lambda_{0}<0, \lambda_{1}=\lambda_{2}=0\right\}, \\
& S_{I}^{B}:=\left\{\lambda \in \mathbb{R}^{n+3} \mid \lambda_{0}>0, \lambda_{1}=\lambda_{2}=0\right\} .
\end{aligned}
$$

Following our notation, we say system (1.4) has a weak center of type $I_{A}$ (resp., type $I_{B}$ ) if the system is nonlinear and $\lambda \in S_{I}^{A}$ (resp., $\lambda \in S_{I}^{B}$ ). We consider weak centers of type $I_{A}$ and type $I_{B}$ separately. Obviously, $p_{2}>0$ for $b_{0}<0$ by Lemma 3.1. Thus, we have the following theorem.

THEOREM 3.2. A weak center of type $I_{A}$ has order 0 and no local critical periods can bifurcate from a weak center of type $I_{A}$.

Now, we discuss weak centers of type $I_{B}$. In this case, the system has the same form as (3.1) with $b_{0}>0$. We have the following lemma.

LEMmA 3.3. Suppose $b_{0}>0$ in (3.1). Then, if $b_{0}=(4 / 9) a_{1}^{2}$ and $a_{2}=\cdots=a_{n}=0$, the origin is an isochronous center.

Proof. Since $b_{0}>0$, the assumption of Lemma 3.3 implies that $a_{1} \neq 0$. Perform coordinate transformation $(x, y) \mapsto\left((2 / 3) a_{1} x,(2 / 3) a_{1} y\right)$, system (3.1) becomes

$$
\dot{x}=-y+\frac{3}{2} x^{2}, \quad \dot{y}=x+x^{3} .
$$

It can be linearized by the change of coordinates $(u, v)=\left(-2 x /\left(x^{2}-2 y-2\right),\left(x^{2}-2 y\right) /\right.$ $\left.\left(x^{2}-2 y-2\right)\right)$ and has first integral $F(x, y)=-4\left(2 x^{2}-2 y-1\right) /\left(x^{2}-2 y-2\right)^{2}$. By [8, Theorem 3.2], the origin is an isochronous center.

To simplify the computation, we scale system (3.1) so that $b_{0}=1$. Then, system (3.1) has the form

$$
\dot{x}=-y+\sum_{k=1}^{n} a_{k} x^{2 k}, \quad \dot{y}=x+x^{3} .
$$

LEMMA 3.4. If $2 \leq k \leq n$, then the period coefficient $p_{2 k}$ of system (3.4) has the form $p_{2 k}=\beta a_{1} a_{k}+q\left(a_{1}, \ldots, a_{k-1}\right)$, where $\beta \neq 0$ is a constant and $q\left(a_{1}, \ldots, a_{k-1}\right) \in$ $\mathbb{R}\left[a_{1}, \ldots, a_{k-1}\right]$.

Proof. The expansion (2.4) for system (3.4) is given by

$$
\begin{aligned}
\frac{d r}{d \theta}= & \frac{\sum_{k=1}^{n}\left(a_{k} \cos ^{2 k+1} \theta\right) r^{2 k}+\left(\cos ^{3} \theta \sin \theta\right) r^{3}}{1-\sum_{k=1}^{n}\left(a_{k} \cos ^{2 k} \theta \sin \theta\right) r^{2 k-1}+\left(\cos ^{4} \theta\right) r^{2}} \\
= & {\left[\sum_{k=1}^{n}\left(a_{k} \cos ^{2 k+1} \theta\right) r^{2 k}+\left(\cos ^{3} \theta \sin \theta\right) r^{3}\right] } \\
& \cdot\left[1+\sum_{m=1}^{\infty}(-1)^{m}\left(\sum_{k=1}^{n}\left(a_{k} \cos ^{2 k} \theta \sin \theta\right) r^{2 k-1}+\left(\cos ^{4} \theta\right) r^{2}\right)^{m}\right] .
\end{aligned}
$$


Similarly, we have

$$
\frac{d t}{d \theta}=1+\sum_{m=1}^{\infty}(-1)^{m}\left[\sum_{k=1}^{n}\left(a_{k} \cos ^{2 k} \theta \sin \theta\right) r^{2 k-1}+\left(\cos ^{4} \theta\right) r^{2}\right]^{m}
$$

Obviously, for any $j \leq 2(k-1)$, where $2 \leq k \leq n$, the coefficients $A_{j}$ and $B_{j}$ in the corresponding expansion (2.5) are independent of $a_{k}$.

On the other hand, $B_{1}=a_{1} \sin \theta \cos ^{2} \theta$ and for $2 \leq k \leq n$,

$$
\begin{gathered}
A_{2 k}=a_{k} \cos ^{2 k+1} \theta+\tilde{A}_{2 k}, \quad B_{2 k-1}=a_{k} \cos ^{2 k} \theta \sin \theta+\tilde{B}_{2 k-1}, \\
B_{2 k}=2 a_{1} a_{k} \cos ^{2 k+2} \theta \sin ^{2} \theta+\tilde{B}_{2 k},
\end{gathered}
$$

where $\tilde{A}_{2 k}, \tilde{B}_{2 k-1}, \tilde{B}_{2 k}$ are polynomials only depending on $a_{1}, \ldots, a_{k-1}$. Furthermore, from (2.5) and (2.7), it is clear that $u_{1}, \ldots, u_{2 k-1}$ only depend on $a_{1}, \ldots, a_{k-1} \cdot u_{2 k}$ satisfies the following initial value problem:

$$
\frac{d u_{2 k}}{d \theta}=A_{2 k} u_{1}^{2 k}+D_{u_{2 k}}, \quad u_{2 k}(0, \lambda)=0,
$$

where $D_{u_{2 k}}$ only depends on $A_{1}, \ldots, A_{2 k-1}$ and $u_{1}, \ldots, u_{2 k-1}$. Solving (3.8) yields $u_{2 k}=$ $a_{k} P_{u_{2 k}}(\cos \theta, \sin \theta)+\tilde{u}_{2 k}$, where $P_{u_{2 k}}(\cos \theta, \sin \theta)$ is a polynomial of $\cos \theta$ and $\sin \theta$ that, independent of $a_{1}, \ldots, a_{n}, \tilde{u}_{2 k}$, is a polynomial only depending on $a_{1}, \ldots, a_{k-1}$. Hence, the period coefficient $p_{2 k}$ can be computed as follows:

$$
p_{2 k}(\lambda)=\int_{0}^{2 \pi}\left(B_{1} u_{2 k}+(2 k-1) B_{2 k-1} u_{1}^{2 k-2} u_{2}+B_{2 k} u_{1}^{2 k}+\tilde{B}_{u}\right) d \theta
$$

where $\tilde{B}_{u}$ only depends on $B_{2}, \ldots, B_{2 k-2}$ and $u_{1}, \ldots, u_{2 k-1}$. Thus, $p_{2 k}$ has the form $p_{2 k}=$ $\beta a_{1} a_{k}+q\left(a_{1}, \ldots, a_{k-1}\right)$, where $\beta \neq 0$ is a constant and $q\left(a_{1}, \ldots, a_{k-1}\right) \in \mathbb{R}\left[a_{1}, \ldots, a_{k-1}\right]$.

Direct computation of period coefficients using the Gröbner base package of Maple for $n \leq 8$ suggests the following Lemma which is proved rigorously.

LEMMA 3.5. For system (3.4), the period coefficients $p_{2 k}(k>1)$, reduced modulo the ideal generated by $p_{2}, \ldots, p_{2 k-2}$ and omitting the constant factor, are given by $p_{2 k}=a_{1} a_{k}$ for $1<k \leq n$ and $p_{2 k}=0$ for $k>n$. In particular, $p_{2 k} \in\left(p_{2}, \ldots, p_{2 n}\right)$, the ideal of the polynomial ring $\mathbb{R}\left[a_{1}, a_{2}, \ldots, a_{n}\right]$.

Proof. We prove Lemma 3.5 by induction on $k$. By direct computation, we find that $p_{4}$ modulo the ideal generated by $p_{2}$ is given by $p_{4}=(2 \pi / 3) a_{1} a_{2}$. Thus, Lemma 3.5 is true for $k=2$.

Now, assume that $2 \leq k \leq n$ and $p_{4}, \ldots, p_{2 k-2}$, with each reduced modulo the ideal generated by the previous coefficients, and omitting the constant factor are given by $p_{2 j}=a_{1} a_{j}(j=2, \ldots, k-1)$. By Lemma $3.4, p_{2 k}=\beta a_{1} a_{k}+q\left(a_{1}, \ldots, a_{k-1}\right)$, where $\beta \neq 0$ is a constant and $q\left(a_{1}, \ldots, a_{k-1}\right) \in \mathbb{R}\left[a_{1}, \ldots, a_{k-1}\right]$. 
Because $p_{2}$ is a quadratic polynomial of $a_{1}, q\left(a_{1}, \ldots, a_{k-1}\right)$ can be written into the form $q\left(a_{1}, \ldots, a_{k-1}\right)=q_{0}\left(a_{1}, \ldots, a_{k-1}\right) p_{2}+a_{1} q_{1}\left(a_{2}, \ldots, a_{k-1}\right)+q_{2}\left(a_{2}, \ldots, a_{k-1}\right)$. Thus, we have

$$
p_{2 k}=\beta a_{1} a_{k}+q_{0}\left(a_{1}, \ldots, a_{k-1}\right) p_{2}+a_{1} q_{1}\left(a_{2}, \ldots, a_{k-1}\right)+q_{2}\left(a_{2}, \ldots, a_{k-1}\right) .
$$

By Lemma 3.3, when $p_{2}=0$ and $a_{2}=\cdots=a_{k}=0, p_{2 k}=0$. Thus, $q_{1}(0, \ldots, 0)=$ $q_{2}(0, \ldots, 0)=0$. Hence, they have the following form:

$$
\begin{aligned}
& q_{1}\left(a_{2}, \ldots, a_{k-1}\right)=\sum_{j=2}^{k-1} a_{j} S_{j}\left(a_{2}, \ldots, a_{k-1}\right), \\
& q_{2}\left(a_{2}, \ldots, a_{k-1}\right)=\sum_{j=2}^{k-1} a_{j} R_{j}\left(a_{2}, \ldots, a_{k-1}\right),
\end{aligned}
$$

where $S_{j}\left(a_{2}, \ldots, a_{k-1}\right)$ and $R_{j}\left(a_{2}, \ldots, a_{k-1}\right)$ are polynomials of $a_{2}, \ldots, a_{k-1}$. By induction hypothesis, $a_{1} a_{j}=p_{2 j}(j=2, \ldots, k-1)$. Thus, (3.10) becomes

$$
p_{2 k}=\beta a_{1} a_{k}+q_{0} p_{2}+\sum_{j=2}^{k-1} p_{2 j} S_{j}+\sum_{j=2}^{k-1} a_{j} R_{j}
$$

On the other hand, we have $\mu p_{2}+\gamma a_{1}^{2}=1$, where $\mu=-4 / 3 \pi, \gamma=4 / 9$. Therefore, by induction hypothesis, for each $a_{j}(2 \leq j \leq k-1)$, we have

$$
a_{j}=\mu a_{j} p_{2}+\gamma a_{1}\left(a_{1} a_{j}\right)=\mu a_{j} p_{2}+\gamma a_{1} p_{2 j}
$$

Substituting (3.13) into (3.12) yields

$$
p_{2 k}=\beta a_{1} a_{k}+q_{0} p_{2}+\sum_{j=2}^{k-1} p_{2 j} S_{j}+\sum_{j=2}^{k-1}\left(\mu a_{j} p_{2}+\gamma a_{1} p_{2 j}\right) R_{j} .
$$

Thus, $p_{2 k}$, reduced modulo $\left(p_{2}, \ldots, p_{2(k-1)}\right)$ and omitting the constant factor, is $a_{1} a_{k}$. This completes the proof of Lemma 3.5 for $1<k \leq n$.

Now, assume that $k>n$. Then, $p_{2 k}$ has the form $p_{2 k}=V_{0}\left(a_{1}, \ldots, a_{n}\right) p_{2}+a_{1} V_{1}\left(a_{2}, \ldots\right.$, $\left.a_{n}\right)+V_{2}\left(a_{2}, \ldots, a_{n}\right)$, where $V_{0}, V_{1}, V_{2}$ are polynomials. By Lemma 3.3, when $p_{2}=0$ and $a_{2}=\cdots=a_{n}=0, p_{2 k}=0$. Thus, for $j=1,2, V_{j}(0, \ldots, 0)=0$. Using the same method as above and the result about $p_{2}, \ldots, p_{2 n}$ we just proved, it is straightforward to show that $p_{2 k}$, reduced modulo the ideal generated by $p_{2}, \ldots, p_{2 n}$, is zero. Hence, for any $k \geq 1$, $p_{2 k} \in\left(p_{2}, \ldots, p_{2 n}\right)$.

Lemma 3.5 describes the simple structure of the period coefficients which enables us to prove the following theorem. 
THEOREM 3.6. If the origin is a weak center of type $I_{B}$, then it is an isochronous center of (3.1) if and only if $b_{0}=(4 / 9) a_{1}^{2}$ and $a_{2}=\cdots=a_{n}=0$. If the origin is a weak center of finite order, then its order is at most $n-1$, at most $n-1$ local critical periods can bifurcate and there are perturbations with exactly $j$ critical periods for each $j \leq n-1$. Moreover, at most $n-1$ local critical periods can bifurcate from the isochronous center and there are perturbations to produce the maximum number of critical periods. Here, all perturbations of parameters are within $S_{I}^{B}$.

Proof. We first prove that the origin is an isochronous center if and only if $b_{0}=$ (4/9) $a_{1}^{2}$ and $a_{2}=\cdots=a_{n}=0$. The sufficiency of the condition has been proved in Lemma 3.3. Now, assume that the origin is an isochronous center. By Lemma 3.1, $p_{2}=0$ if and only if $b_{0}=(4 / 9) a_{1}^{2}>0$. Perform coordinate transformation $(x, y) \mapsto$ $\left((2 / 3) a_{1} x,(2 / 3) a_{1} y\right)$, hence system (3.1) becomes

$$
\dot{x}=-y+\sum_{k=1}^{n} \tilde{a}_{k} x^{2 k}, \quad \dot{y}=x+x^{3} \text {, }
$$

where $\tilde{a}_{k}=\left(3 / 2 a_{1}\right)^{2 k-1} a_{k}$. System (3.15) has the same form as (3.4). Hence, by Lemma $3.5, p_{4}=\cdots=p_{2 n}=0$ if and only if $\tilde{a}_{2}=\cdots=\tilde{a}_{n}=0$. This implies that $a_{2}=\cdots=$ $a_{n}=0$. Thus, the necessity of the condition is also proved.

To discuss the local critical periods, note that $b_{0}>0$, we can scale system (3.1) so that $b_{0}=1$. It suffices to consider (3.4) only.

If the origin is a weak center of finite order and $p_{2}=0$, then $a_{1}^{2}=9 / 4$ and there must be an integer $k$, such that $1<k \leq n$ and $a_{2}=\cdots=a_{k-1}=0$ and $a_{k} \neq 0$. Thus, by Lemma $3.5, p_{2}=\cdots=p_{2(k-1)}=0, p_{2 k}=a_{1} a_{k} \neq 0$ (with a nonzero constant factor omitted). That is, the origin is a weak center of order $k-1$. Hence, the maximum order of the weak center is $n-1$ and at most $n-1$ local critical periods can bifurcate.

Now, assume that the origin is a weak center of order $n-1$. Then, $a_{1}^{2}=9 / 4, a_{2}=$ $\cdots=a_{n-1}=0$, and $a_{n} \neq 0$. Let $v_{*}=\left(a_{1}, 0, \ldots, 0, a_{n}\right)$, where $a_{1}=3 / 2$ or $-3 / 2$. Let $a_{1}^{\prime}$ be $3 / 2$ or $-3 / 2$. It is straightforward to see that the algebraic surfaces $p_{2(n-2)}\left(a_{1}^{\prime}\right.$, $\left.0, \ldots, 0, a_{n-2}^{\prime}, a_{n-1}^{\prime}, a_{n}^{\prime}\right)=0$ and $p_{2(n-1)}\left(a_{1}^{\prime}, 0, \ldots, 0, a_{n-2}^{\prime}, a_{n-1}^{\prime}, a_{n}^{\prime}\right)=0$ intersect transversally at their common roots for $\left(a_{n-2}^{\prime}, a_{n-1}^{\prime}\right) \in(-\infty, \infty) \times(-\infty, \infty)$. In fact, from the expressions for the period coefficients given in Lemma 3.5, the determinant of the Jacobian matrix of $p_{2(n-2)}\left(a_{1}^{\prime}, 0, \ldots, 0, a_{n-2}^{\prime}, a_{n-1}^{\prime}, a_{n}^{\prime}\right)$ and $p_{2(n-1)}\left(a_{1}^{\prime}, 0, \ldots, 0, a_{n-2}^{\prime}, a_{n-1}^{\prime}\right.$, $\left.a_{n}^{\prime}\right)$ is given by $a_{1}^{\prime 2}$, which is not zero. This guarantees that in the neighborhood of $v_{*}$, there exists a perturbation $v^{\prime}$ such that $p_{2(n-2)}\left(v^{\prime}\right) p_{2(n-1)}\left(v^{\prime}\right)<0$ with $p_{2 n}\left(v^{\prime}\right) \neq 0$. This implies that, in the neighborhood of such perturbation, we may choose $\tilde{v}_{*}$ such that the system satisfies the condition $\left(\mathscr{P}_{k}\right)$ with $k=n-1$. Thus, by the finite-order bifurcation theorem, there are perturbations with exactly $j$ critical periods for each $j \leq n-1$.

Denote by $\tilde{v}_{*}=\left(\delta+\epsilon_{1}, \epsilon_{2}, \ldots, \epsilon_{n}\right)$ the perturbation of the isochronous center, where $\delta$ is $3 / 2$ or $-3 / 2$. Denote by $\tilde{p}_{2 k}\left(\tilde{v}_{*}\right)$ the perturbed period coefficients. Using the same method as in the proof of Lemma 3.5, we find the perturbed period coefficients, with each reduced modulo the ideal generated by the previous coefficients, are given by $\tilde{p}_{2}\left(\tilde{v}_{*}\right)=(\pi / 3) \epsilon_{1}\left(2 \delta+\epsilon_{1}\right), \tilde{p}_{2 k}\left(\tilde{v}_{*}\right)=\epsilon_{k}\left(\delta+\epsilon_{1}\right)$ for $2 \leq k \leq n, \tilde{p}_{2 k}\left(\tilde{v}_{*}\right)=0$ for $k>n$. 
Clearly, for any $k \geq 1, \tilde{p}_{2 k}\left(\tilde{v}_{*}\right) \in\left(\tilde{p}_{2}\left(\tilde{v}_{*}\right), \ldots, \tilde{p}_{2 n}\left(\tilde{v}_{*}\right)\right)$, the ideal of the Noetherian ring $\mathbb{R}\left\{a_{1}, \ldots, a_{n}\right\}_{v_{*}}$ of convergent power series at $v_{*}=(\delta, 0, \ldots, 0)$. Thus, by the isochrone bifurcation theorem, at most $n-1$ local critical periods can bifurcate from the isochronous center. Similar to the argument in the previous paragraph, the maximum number can be reached.

4. Weak center of type $I I$. A weak center of type $I I$ corresponds to the following system:

$$
\dot{x}=-y+\sum_{k=1}^{n} a_{k}\left(x^{2}+b_{1} x^{3}+b_{2} x^{4}\right)^{k}, \quad \dot{y}=x+\frac{3}{2} b_{1} x^{2}+2 b_{2} x^{3} .
$$

Executing our Maple program yields the following lemma.

LEMMA 4.1. The period coefficient $p_{2}$ for (4.1) is given by $p_{2}=(\pi / 24)\left(8 a_{1}^{2}+45 b_{1}^{2}-\right.$ $\left.36 b_{2}\right)$.

We split $S_{I I}$ into 3 subsets as $S_{I I}=S_{I I}^{A} \cup S_{I I}^{B} \cup S_{I I}^{C}$, where

$$
\begin{aligned}
& S_{I I}^{A}:=\left\{\lambda \in \mathbb{R}^{n+3} \mid \lambda_{0}=0, \lambda_{2} \leq 0\right\}, \\
& S_{I I}^{B}:=\left\{\lambda \in \mathbb{R}^{n+3} \mid \lambda_{0}=\lambda_{1}=0, \lambda_{2}>0\right\}, \\
& S_{I I}^{C}:=\left\{\lambda \in \mathbb{R}^{n+3} \mid \lambda_{0}=0, \lambda_{1} \neq 0, \lambda_{2}>0\right\} .
\end{aligned}
$$

We say system (4.1) has a weak center of type $I I_{A}$ (resp., type $I I_{B}$ or type $I I_{C}$ ) if the system is nonlinear and $\lambda \in S_{I I}^{A}$ (resp., $\lambda \in S_{I I}^{B}$ or $\lambda \in S_{I I}^{C}$ ). We immediately have the following theorem.

THEOREM 4.2. A weak center of type $I I_{A}$ cannot be an isochronous center and no local critical periods can bifurcate from a weak center of type $I I_{A}$.

Proof. If $b_{2} \leq 0$ and $b_{1}^{2}+b_{2}^{2} \neq 0$, then $p_{2}>0$ by Lemma 4.1 and the order of the weak center is 0 . Thus, no local critical periods can bifurcate by finite order bifurcation theorem. If $b_{1}=b_{2}=0$, then the weak center is of type $I_{A}$, there are no local critical periods that can bifurcate by Theorem 3.2.

Thus, in the following we only need to discuss weak centers of types $I I_{B}$ and $I I_{C}$.

4.1. Weak center of type $I I_{B}$. For a weak center of type $I I_{B}$, we can scale system (4.1) so that $b_{2}=1 / 2$. Thus, we only need to consider the following system:

$$
\dot{x}=-y+\sum_{k=1}^{n} a_{k}\left(x^{2}+\frac{x^{4}}{2}\right)^{k}, \quad \dot{y}=x+x^{3} .
$$

We first prove the following lemma.

LEMMA 4.3. A weak center of type $I I_{B}$ cannot be an isochronous center. 
Proof. Clearly, system (4.3) can be written into the form

$$
\dot{x}=-y+\sum_{k=1}^{n} c_{k} x^{2 k}, \quad \dot{y}=x+x^{3},
$$

where

$$
c_{k}=\sum_{j=0}^{[k / 2]} \frac{1}{2^{j}} \frac{(k-j) !}{j !(k-2 j) !} a_{k-j} .
$$

System (4.4) has the same form as (3.4). By Theorem 3.6, if the origin is an isochronous center, then $c_{2}=\cdots=c_{2 n}=0$ and $c_{1}^{2}=9 / 4$. But $c_{2}=\cdots=c_{2 n}=0$ implies that $a_{1}=$ $a_{2}=\cdots=a_{n}=0$. Hence, $c_{1}=0$. This is a contradiction.

The following Lemma characterizes the ideal generated by the period coefficients.

LEMMA 4.4. For system (4.3), the period coefficients $p_{2 k}(k>1)$, reduced modulo the ideal generated by $p_{2}, \ldots, p_{2 k-2}$ and omitting the constant factor, are given by $p_{2 k}=$ $a_{1} a_{k}+\alpha_{k}$ for $1<k \leq n, p_{2(n+1)}=\alpha_{n+1}$ and $p_{2 k}=0$ for $k>n+1$, where $\alpha_{1}, \ldots, \alpha_{n+1}$ are nonzero constants. In particular, $p_{2 k} \in\left(p_{2}, \ldots, p_{2(n+1)}\right)$, the ideal of the polynomial ring $\mathbb{R}\left[a_{1}, a_{2}, \ldots, a_{n}\right]$.

Proof. We first prove the result about $p_{2 k}$ for $1<k \leq n$ by induction on $k$. Direct computation yields $p_{2}=(\pi / 12)\left(4 a_{1}^{2}-9\right), p_{4}$ reduced modulo the ideal generated by $p_{2}$ and omitting the constant factor is $p_{4}=a_{1} a_{2}+9 / 8$.

Now, assume that $2 \leq k \leq n$ and $p_{4}, \ldots, p_{2(k-1)}$ with each reduced modulo the ideal generated by previous coefficients and omitting the constant factor, are given by $p_{2 j}=$ $a_{1} a_{j}+\alpha_{j}(j=2, \ldots, k-1)$, where $\alpha_{j}$ is nonzero constant. Similar to the proof of Lemma $3.4, p_{2 k}$ has the form $p_{2 k}=\beta a_{1} a_{k}+R\left(a_{1}, \ldots, a_{k-1}\right)$, where $\beta$ is a nonzero constant and $R\left(a_{1}, \ldots, a_{k-1}\right)$ is a polynomial. Since $p_{2}=(\pi / 12)\left(4 a_{1}^{2}-9\right)$, we can write $R\left(a_{1}, \ldots, a_{k-1}\right)$ $=R_{1}\left(a_{1}, \ldots, a_{k-1}\right) p_{2}+a_{1} R_{2}\left(a_{2}, \ldots, a_{k-1}\right)+R_{3}\left(a_{2}, \ldots, a_{k-1}\right)$, where $R_{1}, R_{2}, R_{3}$ are polynomials. We claim that $R_{2}(0, \ldots, 0)=0$.

In fact, if we set $a_{2}=\cdots=a_{n}=0$, then system (4.3) has the form

$$
\dot{x}=-y+a_{1}\left(x^{2}+\frac{x^{4}}{2}\right), \quad \dot{y}=x+x^{3} .
$$

Let $T\left(\xi, a_{1}\right)$ be the minimum period of the closed orbit of (4.6) passing through $(\xi, 0)$. Then, $T\left(\xi,-a_{1}\right)$ is the minimum period of the closed orbit of the following system passing through $(\xi, 0)$ :

$$
\dot{x}=-y-a_{1}\left(x^{2}+\frac{x^{4}}{2}\right), \quad \dot{y}=x+x^{3} .
$$

But (4.7) can be transformed to system (4.6) by the scaling $(x, y) \mapsto(-x,-y)$ and this scaling does not change the period of the closed orbit passing through $(\xi, 0)$. Thus, $T\left(\xi, a_{1}\right)=T\left(\xi,-a_{1}\right)$. Hence, when $a_{2}=\cdots=a_{n}=0$, the period coefficients are 
functions of $a_{1}^{2}$. Thus, $R\left(a_{1}, 0, \ldots, 0\right)=R_{1}\left(a_{1}, 0, \ldots, 0\right) p_{2}+a_{1} R_{2}(0, \ldots, 0)+R_{3}(0, \ldots, 0)$ is a polynomial of $a_{1}^{2}$. Since $p_{2}=(\pi / 12)\left(4 a_{1}^{2}-9\right)$, it is clear that $R_{1}\left(a_{1}, 0, \ldots, 0\right)$ does not have terms of $a_{1}$ with odd degree. Thus, $R_{2}(0, \ldots, 0)=0$.

Hence, $R_{2}$ has the form

$$
R_{2}\left(a_{2}, \ldots, a_{k-1}\right)=a_{2} S_{2}\left(a_{2}, \ldots, a_{k-1}\right)+\cdots+a_{k-1} S_{k-1}\left(a_{2}, \ldots, a_{k-1}\right),
$$

where $S_{2}, \ldots, S_{k-1}$ are polynomials. Therefore, $p_{2 k}$ can be written as

$$
p_{2 k}=\beta a_{1} a_{k}+R_{1} p_{2}+\sum_{i=2}^{k-1} a_{1} a_{i} S_{i}\left(a_{2}, \ldots, a_{k-1}\right)+R_{3}\left(a_{2}, \ldots, a_{k-1}\right) .
$$

By induction assumption, $a_{1} a_{i}=p_{2 i}-\alpha_{i}$, so (4.9) can be written in the form

$$
p_{2 k}=\beta a_{1} a_{k}+R_{1} p_{2}+\sum_{i=2}^{k-1} S_{i} p_{2 i}+Q\left(a_{2}, \ldots, a_{k-1}\right),
$$

where $Q\left(a_{2}, \ldots, a_{k-1}\right)$ is a polynomial and can be written as $Q\left(a_{2}, \ldots, a_{k-1}\right)=\kappa+$ $Q_{2}\left(a_{2}, \ldots, a_{k-1}\right)$, where $Q_{2}$ is a polynomial such that $Q_{2}(0, \ldots, 0)=0$ and $\kappa$ is a constant.

On the other hand, $p_{2}=(\pi / 12)\left(4 a_{1}^{2}-9\right)$, which implies that $\mu p_{2}+\gamma a_{1}^{2}=1$, where $\mu=-4 / 3 \pi, \gamma=4 / 9$. Therefore, by induction hypothesis, for each $a_{j}(2 \leq j \leq k-1)$, we have

$$
a_{j}=\mu a_{j} p_{2}+\gamma a_{1}\left(a_{1} a_{j}\right)=\mu a_{j} p_{2}+\gamma a_{1} p_{2 j}+\tau_{j} a_{1}
$$

where $\tau_{j}=-\gamma \alpha_{j}$ is a constant. Thus, for any monomial $a_{2}^{j_{2}} \cdots a_{k-1}^{j_{k-1}}$ with $m:=j_{2}+$ $\cdots+j_{k-1}>0$, after reducing modulo the ideal generated by $p_{2}, \ldots, p_{2(k-1)}$, it equals $\tau a_{1}^{m}$ for some constant $\tau$. Since $Q_{2}\left(a_{2}, \ldots, a_{k-1}\right)$ is a combination of such type of monomials, it is clear that, after reducing modulo the ideal generated by $p_{2}, \ldots, p_{2(k-1)}$, $Q_{2}\left(a_{2}, \ldots, a_{k-1}\right)=\tilde{Q}\left(a_{1}\right)$, a polynomial of $a_{1}$. Combining this with (4.10), it is obvious that, after reducing modulo the ideal generated by $p_{2}, \ldots, p_{2(k-1)}, p_{2 k}=\beta a_{1} a_{k}+\tilde{Q}\left(a_{1}\right)+$ $\kappa$. Similar to the argument above, $\tilde{Q}\left(a_{1}\right)$ is a polynomial of $a_{1}^{2}$. Hence, it can be written in the form $\tilde{Q}\left(a_{1}\right)=\hat{Q}\left(a_{1}\right) p_{2}+\iota$, where $\iota$ is a constant and $\hat{Q}\left(a_{1}\right)$ is a polynomial. Therefore, the reduced $p_{2 k}$ is $p_{2 k}=\beta a_{1} a_{k}+\iota+\kappa=\beta\left(a_{1} a_{k}+\alpha_{k}\right)$, where $\alpha_{k}=(\iota+\kappa) / \beta$ is a constant. If we omit the constant factor, then $p_{2 k}=a_{1} a_{k}+\alpha_{k}$. It is trivial to show that $\alpha_{k}$ is nonzero: just set $p_{2}=0$ and $a_{2}=\cdots=a_{n}=0$, simple computation shows that $p_{4}, \ldots, p_{2 n}$, which are multiples of $\alpha_{2}, \ldots, \alpha_{n}$, are all nonzeros.

Now consider $p_{2(n+1)}$, it can be written as

$$
p_{2(n+1)}=V_{1}\left(a_{1}, \ldots, a_{n}\right) p_{2}+a_{1} V_{2}\left(a_{2}, \ldots, a_{n}\right)+V_{3}\left(a_{2}, \ldots, a_{n}\right),
$$


where $V_{1}, V_{2}, V_{3}$ are polynomials. Following the same reasoning as above, we can prove that the reduced $p_{2(n+1)}$ is a nonzero constant $\alpha_{n+1}$. Since $p_{2(n+1)}$ reducing modulo the ideal generated by $p_{2}, \ldots, p_{2 n}$ is a nonzero constant, it is clear that whenever $k>n+1$, $p_{2 k}$ is reduced to zero. It follows that $p_{2 k} \in\left(p_{2}, \ldots, p_{2(n+1)}\right)$ for any $k \geq 1$. The lemma is thus proved.

From Lemma 4.4, we have the following theorem.

THEOREM 4.5. If the origin is a weak center of type $I I_{B}$, then it cannot be an isochronous center and it is a weak center of order at most $n$. At most $n$ local critical periods can bifurcate from the weak center of order $n$ and there are perturbations to produce exactly $j$ critical periods for each $j \leq n$. Here all perturbations of parameters are within $S_{I I}^{B}$.

Proof. For a weak center of type $I I_{B}$, it suffices to consider system (4.3). We have proved in Lemma 4.3 that the origin cannot be an isochronous center. From Lemma 4.4, it is easy to see that the origin is a weak center of order at most $n$. Now, set $a_{1}=3 / 2$ or $a_{1}=-3 / 2$, then $p_{2}=0$. If we set $a_{k}=-\alpha_{k} / a_{1}$ for $2 \leq k \leq n$, where $\alpha_{k}$ is the nonzero constant stated in Lemma 4.4 , then we have $p_{2}=\cdots=p_{2 n}=0$ and $p_{2(n+1)} \neq 0$. Thus, there exists a weak center of order $n$. By finite order bifurcation theorem, at most $n$ critical periods can bifurcate from the weak center of order $n$.

Denote $v_{*}=\left(\delta,-\alpha_{2} / \delta, \ldots,-\alpha_{n} / \delta\right)$, where $\delta=3 / 2$ or $\delta=-3 / 2$. Then, $p_{2}\left(\nu_{*}\right)=\cdots=$ $p_{2 n}\left(\nu_{*}\right)=0$ and $p_{2(n+1)}\left(\nu_{*}\right) \neq 0$. For any neighborhood $W \subset \mathbb{R}^{n}$ of $v_{*}$, let $v^{\prime} \in W$, $v^{\prime}=\left(a_{1}^{\prime}, \ldots, a_{n}^{\prime}\right)$, such that $p_{2}\left(v^{\prime}\right)=\cdots=p_{2 n-2}\left(v^{\prime}\right)=0$. Then, $p_{2 n}\left(v^{\prime}\right)=a_{1}^{\prime} a_{n}^{\prime}+\alpha_{n}$ and $p_{2 n+2}\left(\nu^{\prime}\right)=\alpha_{n+1}$. Obviously, we can always pick $a_{1}^{\prime}$ near $\delta$ and $a_{n}^{\prime}$ near $-\alpha_{n} / \delta$ such that $p_{2 n}\left(\nu^{\prime}\right) p_{2 n+2}\left(\nu^{\prime}\right)<0$. Thus, the system satisfies condition $\left(\mathscr{P}_{k}\right)$ with $k=n$. by finite order bifurcation theorem, there are perturbations to produce exactly $j$ critical periods for each $j \leq n$.

4.2. Weak center of type $I I_{C}$. For a weak center of type $I I_{C}$, we again scale system (4.1) so that $b_{2}=1 / 2$. So, system (4.1) has the following form:

$$
\dot{x}=-y+\sum_{k=1}^{n} a_{k}\left(x^{2}+b_{1} x^{3}+\frac{x^{4}}{2}\right)^{k}, \quad \dot{y}=x+\frac{3}{2} b_{1} x^{2}+x^{3} .
$$

Clearly, $G(x)=\int_{0}^{x} g(\xi) d \xi=M / 2$. In [6], Christopher and Devlin proved that if $F(x)=$ $f_{1} M+\cdots+f_{r} M^{r}$ and $G(x)=g_{1} M+\cdots+g_{p} M^{p}$, then a necessary condition for the origin to be an isochronous center is that $p=2 r$. From this result, we immediately have the following lemma.

LEMMA 4.6. A weak center of type $I_{C}$ cannot be an isochronous center.

It is much harder to describe the ideal generated by the period coefficients. Based on computation for $n \leq 6$, we believe that for any $k \geq 1$, the period coefficient $p_{2 k} \in$ $\left(p_{2}, \ldots, p_{2(n+2)}\right)$, the ideal of the polynomial ring $\mathbb{R}\left[b_{1}, a_{1}, \ldots, a_{n}\right]$. Although we are unable to rigorously prove this, we are able to estimate the lower bound of the maximum number of local critical periods. First, we have the following lemma. 
LEMMA 4.7. For system (4.13), the period coefficients $p_{2 k}(k \geq 1)$ are polynomials of $b_{1}^{2}$.

Proof. Performing the coordinate change $(x, y) \mapsto(x, y-F(x))$, system (4.13) can be rewritten into the form

$$
\begin{gathered}
\dot{x}=-y, \\
\dot{y}=x+\frac{3}{2} b_{1} x^{2}+x^{3}+\left(2 x+3 b_{1} x^{2}+2 x^{3}\right) \sum_{k=1}^{n} k a_{k}\left(x^{2}+b_{1} x^{3}+\frac{x^{4}}{2}\right)^{k-1} y,
\end{gathered}
$$

where

$$
F(x)=\sum_{k=1}^{n} a_{k}\left(x^{2}+b_{1} x^{3}+\frac{x^{4}}{2}\right)^{k} .
$$

The coordinate change is nonsingular near the origin and it does not change the periods of the closed orbits near the origin.

Let $T\left(\xi, b_{1}, a_{1}, \ldots, a_{n}\right)$ be the minimum period of the closed orbit of (4.13) passing through $(\xi, 0)$. Then, $T\left(\xi,-b_{1}, a_{1}, \ldots, a_{n}\right)$ is the minimum period of the closed orbit of the following system passing through $(\xi, 0)$ :

$$
\begin{gathered}
\dot{x}=-y, \\
\dot{y}=x-\frac{3}{2} b_{1} x^{2}+x^{3}+\left(2 x-3 b_{1} x^{2}+2 x^{3}\right) \sum_{k=1}^{n} k a_{k}\left(x^{2}-b_{1} x^{3}+\frac{x^{4}}{2}\right)^{k-1} y .
\end{gathered}
$$

But (4.16) can be transformed to (4.14) via $(x, y) \mapsto(-x,-y)$. Thus, $T\left(\xi, b_{1}, a_{1}, \ldots, a_{n}\right)=$ $T\left(\xi,-b_{1}, a_{1}, \ldots, a_{n}\right)$. Hence, $T\left(\xi, b_{1}, a_{1}, \ldots, a_{n}\right)$ is a function of $b_{1}^{2}$; namely, the period coefficients are polynomials of $b_{1}^{2}$.

Note that for $b_{1}=0$, system (4.13) is the same as system (4.3). We have the following theorem.

THEOREM 4.8. The maximum number of local critical periods which can bifurcate from a weak center of type $I I_{C}$ is at least $n$.

Proof. Let $T\left(\xi, b_{1}, a_{1}, \ldots, a_{n}\right)$ be the minimum period of the closed orbit passing through $(\xi, 0)$. By Lemma 4.7 ,

$$
T\left(\xi, b_{1}, a_{1}, \ldots, a_{n}\right)=T\left(\xi, 0, a_{1}, \ldots, a_{n}\right)+b_{1}^{2} \tilde{T}\left(\xi, b_{1}^{2}, a_{1}, \ldots, a_{n}\right),
$$

where $\tilde{T}\left(\xi, b_{1}^{2}, a_{1}, \ldots, a_{n}\right)$ is an analytic function. Clearly, $T\left(\xi, 0, a_{1}, \ldots, a_{n}\right)$ is identical to the period function of system (4.3). Let

$$
P\left(\xi, b_{1}, a_{1}, \ldots, a_{n}\right)=T\left(\xi, b_{1}, a_{1}, \ldots, a_{n}\right)-2 \pi,
$$


then

$$
\begin{aligned}
P\left(\xi, b_{1}, a_{1}, \ldots, a_{n}\right) & =P\left(\xi, 0, a_{1}, \ldots, a_{n}\right)+b_{1}^{2} \tilde{T}\left(\xi, b_{1}^{2}, a_{1}, \ldots, a_{n}\right), \\
P_{\xi}\left(\xi, b_{1}, a_{1}, \ldots, a_{n}\right) & =P_{\xi}\left(\xi, 0, a_{1}, \ldots, a_{n}\right)+b_{1}^{2} \tilde{T}_{\xi}\left(\xi, b_{1}^{2}, a_{1}, \ldots, a_{n}\right) .
\end{aligned}
$$

By Theorem 4.5, the function $\xi \mapsto P_{\xi}\left(\xi, 0, a_{1}, \ldots, a_{n}\right)$ can have at most $n$ zeros near $\xi=0$ and there exists $a_{1}^{\prime}, \ldots, a_{n}^{\prime}$ such that $P_{\xi}\left(\xi, 0, a_{1}^{\prime}, \ldots, a_{n}^{\prime}\right)$ has $n$ zeros near $\xi=0$. Furthermore, following exactly the same line as in the proof of the finite order bifurcation theorem given in [3] (i.e., [3, Theorem 2.1]), we may select $a_{1}^{\prime}, \ldots, a_{n}^{\prime}$ and construct the $n$ zeros $\xi_{1}, \ldots, \xi_{n}$ such that $0<\xi_{1}<\cdots<\xi_{n}<\eta$ for some $\eta$ and on each pair of the open intervals $\left(\xi_{i-1}, \xi_{i}\right),\left(\xi_{i}, \xi_{i+1}\right)(1 \leq i \leq n), P_{\xi}\left(\xi, 0, a_{1}^{\prime}, \ldots, a_{n}^{\prime}\right)$ has different signs. Here, we set $\xi_{0}=0$ and $\xi_{n+1}=\eta$. Thus, there exists $\xi_{0}^{\prime}, \ldots, \xi_{n}^{\prime}$ such that $0<\xi_{0}^{\prime}<\cdots<\xi_{n}^{\prime}<\eta$ and for any $1 \leq i \leq n, P_{\xi}\left(\xi_{i-1}^{\prime}, 0, a_{1}^{\prime}, \ldots, a_{n}^{\prime}\right) P_{\xi}\left(\xi_{i}^{\prime}, 0, a_{1}^{\prime}, \ldots, a_{n}^{\prime}\right)<0$. Since $\tilde{T}_{\xi}\left(\xi, b_{1}^{2}, a_{1}^{\prime}, \ldots, a_{n}^{\prime}\right)$ is continuous (actually analytic) on $[0, \eta]$, it is easy to see that for sufficiently small $\left|b_{1}\right|, P_{\xi}\left(\xi_{i-1}^{\prime}, b_{1}, a_{1}^{\prime}, \ldots, a_{n}^{\prime}\right) P_{\xi}\left(\xi_{i}^{\prime}, b_{1}, a_{1}^{\prime}, \ldots, a_{n}^{\prime}\right)<0$. Hence, there exists $\tilde{\xi}_{i} \in\left(\xi_{i-1}^{\prime}, \xi_{i}^{\prime}\right)$ such that $P_{\xi}\left(\tilde{\xi}_{i}, b_{1}, a_{1}^{\prime}, \ldots, a_{n}^{\prime}\right)=0$. So, $P_{\xi}\left(\xi, b_{1}, a_{1}^{\prime}, \ldots, a_{n}^{\prime}\right)$ has at least $n$ zeros.

By finite order bifurcation theorem, no more than $q$ local critical periods can bifurcate from a weak center of order $q$. Hence, we immediately obtain the following corollary to Theorem 4.8.

COROLLARY 4.9. There are weak centers of type $I_{C}$ with order at least $n$.

If our conjecture that $p_{2 k} \in\left(p_{2}, \ldots, p_{2(n+2)}\right)$ is true, then since the origin cannot be an isochronous center, the origin is a weak center of order at most $n$ and there are at most $n$ local critical periods that can bifurcate from the weak center of order $n$. By Theorem 4.8, the maximum number of critical periods can be attained.

\section{Proof of Theorem 1.5}

Proof of Theorem 1.5. By Theorems 3.2, 3.6, 4.2, 4.5, and Lemma 4.6, system (1.4) has a nonlinear isochronous center at the origin if and only if $b_{0}=(4 / 9) a_{0}^{2}$ and $b_{1}=b_{2}=a_{1}=\cdots=a_{n}=0$ and $a_{0} \neq 0$. If $a_{0}=0$, then the origin is a linear isochronous center. For a nonlinear isochronous center, $b_{0} \neq 0$. Since we only consider small perturbation of parameter values, any perturbation of the parameters corresponding to the isochronous center remains in $S_{I}^{B}$. Thus, assertion (1) of Theorem 1.5 is true by Theorem 3.6.

Note that under small perturbation of parameter values, a weak center of type $I_{A}$ (resp., $I_{B}$ ) is still a weak center of type $I_{A}$ (resp., $I_{B}$ ). Thus, assertion (2) of Theorem 1.5 is true by Theorems 3.2 and 3.6. Assertion (3) of Theorem 1.5 is clear by Theorem 4.8.

We remark that a weak center of type $I I$ can be perturbed to become a weak center of type $I$, but this will not increase the number of local critical periods by (2) of Theorem 1.5. 
ACKNOWLEDGMENTS. The author thanks the referees for their useful remarks and suggestions. This work is partially supported by Science Foundation for Young Scholars of Sichuan University, Grant no. 0020105505017.

\section{REFERENCES}

[1] W. A. Albarakati, N. G. Lloyd, and J. M. Pearson, Transformation to Liénard form, Electron. J. Differential Equations 2000 (2000), no. 76, 1-11.

[2] N. N. Bautin, On the number of limit cycles which appear with the variation of coefficients from an equilibrium position of focus or center type, American Math. Soc. Translation 1954 (1954), no. 100, 1-19.

[3] C. Chicone and M. Jacobs, Bifurcation of critical periods for plane vector fields, Trans. Amer. Math. Soc. 312 (1989), no. 2, 433-486.

[4] S.-N. Chow and J. A. Sanders, On the number of critical points of the period, J. Differential Equations 64 (1986), no. 1, 51-66.

[5] C. Christopher, An algebraic approach to the classification of centers in polynomial Liénard systems, J. Math. Anal. Appl. 229 (1999), no. 1, 319-329.

[6] C. Christopher and J. Devlin, On the classification of Liénard systems with amplitudeindependent periods, J. Differential Equations 200 (2004), no. 1, 1-17.

[7] C. Christopher and S. Lynch, Small-amplitude limit cycle bifurcations for Liénard systems with quadratic or cubic damping or restoring forces, Nonlinearity 12 (1999), no. 4, 1099-1112.

[8] P. Mardešić, C. Rousseau, and B. Toni, Linearization of isochronous centers, J. Differential Equations 121 (1995), no. 1, 67-108.

[9] V. G. Romanovski and M. Han, Critical period bifurcations of a cubic system, J. Phys. A 36 (2003), no. 18, 5011-5022.

[10] C. Rousseau and B. Toni, Local bifurcation of critical periods in vector fields with homogeneous nonlinearities of the third degree, Canad. Math. Bull. 36 (1993), no. 4, 473-484.

[11] _ Local bifurcations of critical periods in the reduced Kukles system, Canad. J. Math. 49 (1997), no. 2, 338-358.

[12] M. Sabatini, On the period function of Liénard systems, J. Differential Equations 152 (1999), no. 2, 467-487.

[13] B. Toni, Small amplitude critical periods in a cubic polynomial system, Southwest J. Pure Appl. Math. (1999), no. 2, 1-12.

[14] W. Zhang, X. Hou, and Z. Zeng, Weak centers and bifurcation of critical periods in reversible cubic systems, Comput. Math. Appl. 40 (2000), no. 6-7, 771-782.

[15] Z. F. Zhang, T. R. Ding, W. Z. Huang, and Z. X. Dong, Qualitative Theory of Differential Equations, Translations of Mathematical Monographs, vol. 101, American Mathematical Society, Rhode Island, 1992.

Zhengdong Du: Department of Mathematics, Sichuan University, Chengdu, Sichuan 610064, China

E-mail address: zdu85@yahoo.com 


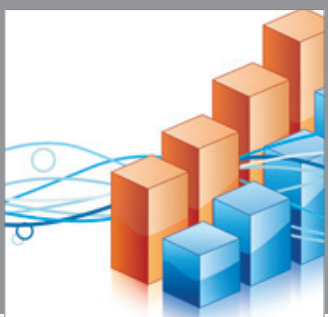

Advances in

Operations Research

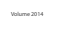

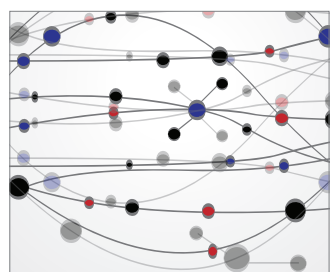

\section{The Scientific} World Journal
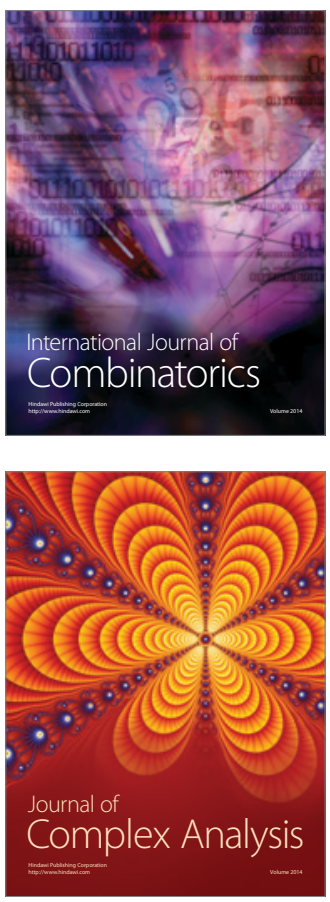

International Journal of

Mathematics and

Mathematical

Sciences
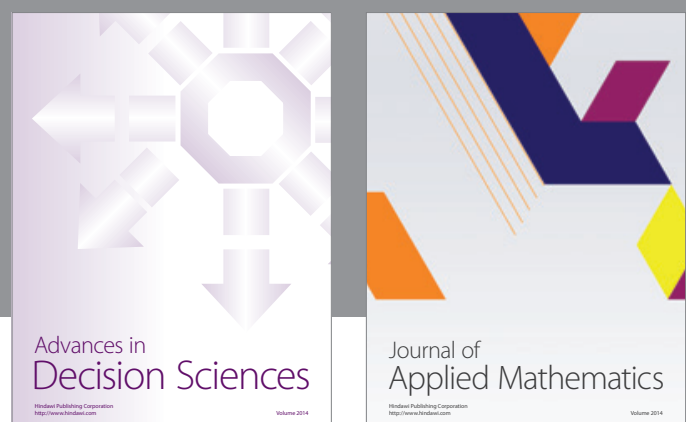

Journal of

Applied Mathematics
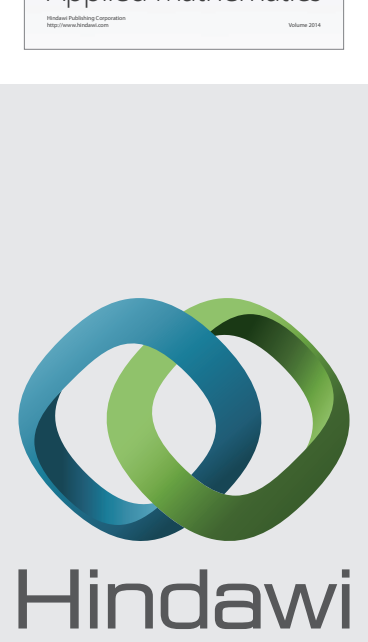

Submit your manuscripts at http://www.hindawi.com
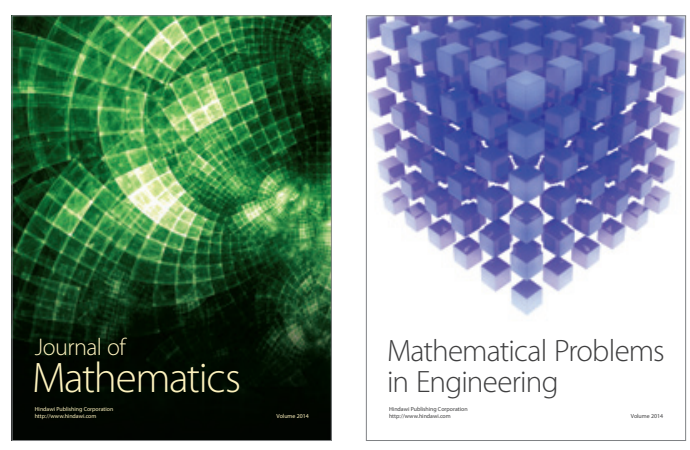

Mathematical Problems in Engineering
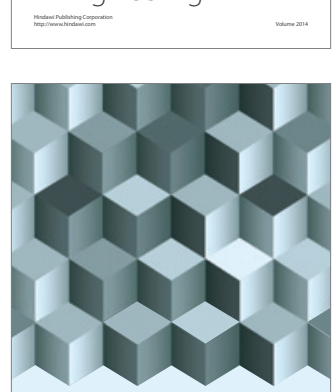

Journal of

Function Spaces
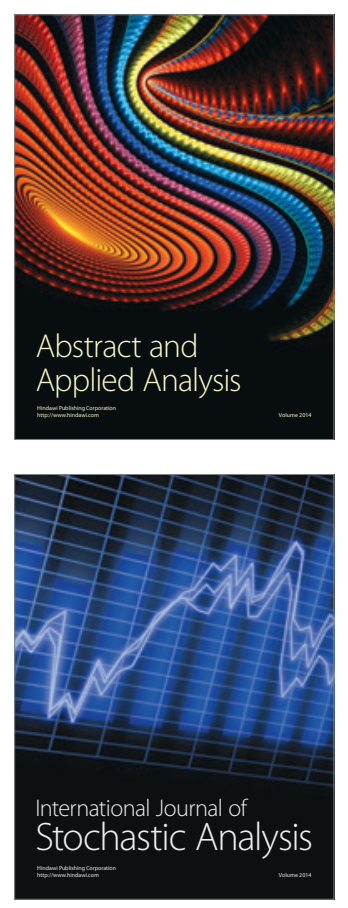

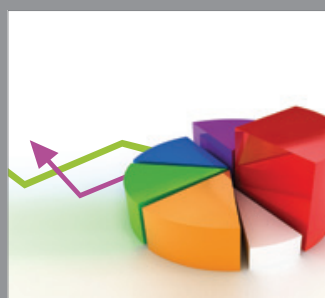

ournal of

Probability and Statistics

Promensencen
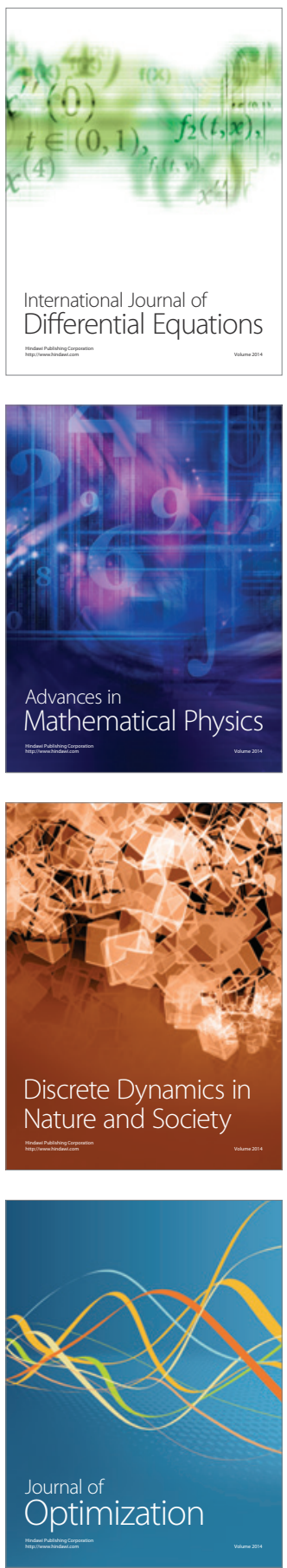